\title{
PERFILES PROFESIONALES Y VALORES RELATIVOS AL TRABAJO
}

\section{PROFESSIONAL PROFILES AND WORK VALUES}

\author{
José Eduardo Moreno \\ Centro Interdisciplinario de Investigaciones en Psicología Matemática y Experimental "Horacio J. A. Rimoldi" \\ (CONICET), Argentina
}

\author{
Antonela Marcaccio \\ Centro Interdisciplinario de Investigaciones en Psicología Matemáticay Experimental "Horacio J. A. Rimoldi" \\ (CONICET), Argentina
}

\begin{abstract}
Resumen: El objetivo del presente trabajo es evaluar la relación existente entre los valores relativos al trabajo y algunos perfiles profesionales (contabilidad y administración, marketing y ventas, y recepción). El instrumento utilizado es la Escala Argentina de Valores Relativos al Trabajo (Moreno \& Marcaccio, 2012) que contiene cuatro subescalas de cinco ítems cada una, a saber: Realización Personal, Reconocimiento Social, Altruismo y Aspiraciones Económicas. La muestra incluye a 425 sujetos (40\% varones y 60\% mujeres) 243 de ellos buscaban trabajar en el área contable, 115 en marketing y 67 en el área de recepción. Se realizó un análisis multivariado de varianza (MANOVA) para determinar las diferencias de medias en las dimensiones de la prueba de valores hacia el trabajo (EVT), se halló que el valor Realización personal es más jerarquizado por los sujetos de marketing. No se observan diferencias según sexo, ni interacción sexo - ocupación.
\end{abstract}

Palabras Clave: valores, trabajo, perfil profesional, adultez

\begin{abstract}
The aim of this research is to evaluate the relationship between work values and some professional profiles (accounting and administration, marketing, and reception). The instrument used is the Work Values Argentine Scale (Moreno \& Marcaccio, 2012). It has four subscales (comprising five items each): Self-realization, Social Acceptance, Altruism and Economic Ambitions. The sample includes 425 cases $(40 \%$ male and $60 \%$ female), 243 subjects pursuing a job in the accounting area, 115 in marketing and 67 in the reception area. A multivariate analysis of variance (MANOVA) was performed to determine the mean differences in the dimensions of the work values scale, we found that marketing sample had the highest media in Self-realization. No differences were observed by sex or interaction of sex - occupation.
\end{abstract}

Keywords: values, work, professional profile, adulthood

\section{Introducción}

El trabajo tiene una significación importante en la vida de las personas adultas y sus familias, ya que es la principal fuente de ingresos en la mayoría de los hogares y es una de las actividades que más organiza la vida cotidiana (Griffa \& Moreno, 2005). El empleo provee a las personas de relaciones y de valoraciones personales (Beccaria \& López, 1997). A medida que un individuo asume o se forma para un cargo, va desarrollando un perfil laboral que le otorga cierta idoneidad para realizar la tarea y para el desempeño eficaz de dicho puesto de trabajo. El trabajo humano supone que las personas pueden realizar su tarea con cierto grado de libertad. Para ello deben conocer sus propias actitudes, intereses, valores, limitaciones y posibilidades, así como también las características de la ocupación a desempeñar y la valoración y demanda social que esa ocupación posee.
Los valores son conceptos o creencias que funcionan como principios-guía. Se considera a los valores como una fuerza que motiva los comportamientos de los individuos (Rokeach, 1973). Dentro de los valores podemos identificar un tipo especial de valores, los relativos al trabajo. Los valores laborales son estándares evaluativos relacionados con el trabajo y el entorno de trabajo. Mediante estos estándares la persona puede evaluar qué le parece correcto, qué le resulta importante y si existe ajuste con sus preferencias (Dose, 1997).

La línea de investigación que los autores de este trabajo vienen desarrollando tiene un interés especial por la evaluación de los valores puestos en juego en determinados ambientes, situaciones y elecciones que se dan durante el ciclo vital de las personas. En este sentido se han construido o adaptado instrumentos de medición que permiten estudiar las complejas relaciones entre el sistema de valores y el sistema motiva- 
cional en diversos campos, incluyendo el ámbito laboral - ocupacional.

El objetivo del presente trabajo es evaluar la relación existente entre los valores relativos al trabajo y algunos perfiles profesionales (contabilidad y administración, marketing y ventas, y recepción).

\section{Marco teórico}

\section{Los valores relativos al trabajo}

Los valores relativos al trabajo son aquellos que guían la vida laboral de las personas y se distinguen cuando una persona debe tomar decisiones ocupacionales o laborales, incluso antes del efectivo desempeño de una ocupación. El concepto de valores hacia el trabajo indica preferencias, más que imperativos morales (Pryor, 1979).

Los valores relativos al trabajo son definidos por Porto y Tamayo (2003, p.146) como "principios o creencias acerca de las metas o recompensas deseadas, jerárquicamente organizados, que las personas buscan por medio del trabajo y que guían su comportamiento, las evaluaciones sobre sus resultados, el contexto y la elección de alternativas laborales". De este modo, los valores relativos hacia el trabajo suponen componentes cognitivos, motivacionales y jerárquicos. Componentes cognitivos porque los valores son un conjunto de creencias acerca de lo que las personas desean, motivacionales porque expresan deseos individuales y jerárquicos porque las personas elaboran jerarquías de valores, en base a la importancia que le atribuyen a cada uno de ellos (Porto \& Tamayo, 2003; Porto, Pilati \& Mendes Taixeira, 2006).

Estudios previos han demostrado el impacto de los valores relativos al trabajo en las organizaciones. Los valores hacia al trabajo influyen tanto en las denominadas variables afectivas en el trabajo, como en el compromiso organizacional (Elizur \& Koslowsky, 2001; Feather \& Rauter, 2004), la satisfacción (Feather \& Rauter, 2004) y el estrés laboral (Siu, 2003; Siu, Spector, Cooper \& Lu, 2005); como en las variables comportamentales tales, como los resultados del trabajo (Takase, Maude \& Manias, 2005) y la intención de rotación (Taris \& Feij, 2001). Por lo tanto, este concepto es especialmente relevante para comprender el mundo del trabajo y se ha convertido en una herramienta útil para elaborar políticas organizacionales y estrategias que favorezcan, tanto el bienestar de los trabajadores, como el de las organizaciones.
A menudo, en psicología organizacional se ha utilizado el modelo de anclas de carrera de Edgar H. Schein $(1978,1993)$. El concepto de anclas de carrera hace referencia a cómo las personas se definen a ellas mismas en relación con el trabajo. El concepto de anclas de carrera incluye los valores básicos, pero además agrega la percepción de los propios talentos y de las propias habilidades, como también de los motivos y necesidades que se relacionan con la carrera. Además, supone que estas anclas se consolidan luego de haber trabajado durante algunos años.

Los valores laborales en ese sentido están presentes antes del efectivo ingreso al mundo laboral y no están directamente relacionados con la evaluación de las propias capacidades del sujeto, ni con sus necesidades (modelo de valores relativo al trabajo).

Si se persiguiera la integración de ambos modelos, los valores serían sólo un componente de las anclas de carrera.

\section{El perfil profesional}

El concepto de perfil profesional tiene cada vez más difusión y se emplea en el ámbito de la educación y de las organizaciones. El perfil profesional, como el perfil del egresado, es un concepto que puede interpretarse con facilidad, intuitivamente, y se utiliza de manera flexible (Arnaz, 1981). El término de perfil profesional suele hacer referencia tanto al producto de una experiencia educativa como a las características que posee un sujeto. Hasta el momento, los diferentes autores no han arribado a una única definición del perfil profesional. El modo en que lo definimos en este trabajo tiene sus antecedentes en el uso que se ha dado en educación y en psicología organizacional.

Se pueden encontrar en la literatura definiciones de perfil profesional que hacen hincapié estrictamente en la formación de profesionales y en el diseño curricular, también pueden encontrarse definiciones que ponen el foco en el tipo de tareas que un sujeto potencialmente pueda realizar o en las competencias y habilidades que posee. Es posible distinguir la existencia de definiciones educativas y laborales del concepto perfil profesional. A grandes rasgos las definiciones laborales comprenden términos como competencias y habilidades, mientras que las definiciones educativas destacan el papel de los conocimientos. Las definiciones de práctica profesional, de perfil profesional y de perfil del 
egresado han contribuido a lo que en este trabajo entendemos por perfil profesional.

Según José Antonio Arnaz $(1981,1996)$, el perfil profesional es una descripción de las características que se requieren del profesional para abarcar y solucionar las necesidades sociales. Un individuo se constituirá como profesional después de haber participado en el sistema de instrucción. Arnaz diferencia perfil profesional de perfil del egresado. Así propone los componentes mínimos que debe contener el perfil del egresado: 1) la especificación de las áreas generales de conocimiento en las cuales deberá adquirir dominio el profesional; 2) la descripción de las tareas, actividades, acciones, etc., que deberá realizar en dichas áreas.; 3) la delimitación de valores y actitudes adquiridas necesarias para su buen desempeño como profesional; y 4) el listado de las destrezas que tiene que desarrollar. De este modo, mediante la inclusión en el diseño curricular, se fomentarán los valores que se necesiten para desempeñarse adecuadamente.

Frida Díaz-Barriga (1999) describe el perfil profesional como un conjunto de conocimientos, habilidades y actitudes que delimitan el ejercicio profesional. El perfil profesional se elabora luego de haber establecido los fundamentos del proceso curricular y forma parte de un proceso, es una etapa dentro de la Metodología de Diseño Curricular. Para la autora es importante definir una visión humanista, científica y social de manera integrada, alrededor de conocimientos, habilidades, destrezas, actitudes y valores. Además, considera que en el caso del perfil profesional es importante incluir la delimitación de las áreas o sectores donde se realizará la labor del egresado, los principales ámbitos de la actividad profesional a realizar y las poblaciones o beneficiarios de su labor profesional (Díaz Barriga , 1993).

Raquel Glazman y María de Ibarrola (1978) definen la noción de práctica profesional que incluye la determinación de las actividades propias de las carreras que se dictan en el nivel universitario. La práctica profesional se compone de dos dimensiones: una se refiere a la actividad por desarrollar e implica la delimitación de un campo de acción y, la otra, se refiere al nivel de comportamiento que alcanza el graduado universitario.

Aunque no define lo que es la práctica profesional, Villarreal (1980) afirma que en parte ésta se constituye en la práctica social, más concretamente en el marco del modo de producción del sistema en que se trabaja, y específicamente en el seno de las relaciones de producción que plantea dicho sistema.

Es responsabilidad de las entidades educativas de todos los niveles desarrollar el perfil profesional de sus graduados que satisfagan las demandas de la sociedad. Los autores de este trabajo, entienden por perfil profesional al conjunto de rasgos y capacidades que pueden estar presentes en personas que no poseen educación formal en un campo del saber, pero sí cuentan con competencias y con conocimientos que las habilitan a desempeñarse en una tarea. Diversos autores han profundizado recientemente en las formaciones que surgen de la propia situación de trabajo, que se refiere a la adquisición de saberes laborales por la vía de interacciones didácticas entre trabajadores, o de los trabajadores con su objeto de trabajo, y en función de la naturaleza misma del sitio de trabajo de que se trate (De Ibarrola, 2004). El perfil profesional se consolida dentro de un proceso histórico y está determinado por el límite y alcance de su campo de acción.

En este estudio se estableció el perfil profesional teniendo en cuenta: la autopercepción de los participantes sobre su adecuación para ocupar un puesto de trabajo, como también la evaluación del curriculum vitae y una entrevista personal, ambas realizadas por un profesional del área de psicología del trabajo.

Se trabajó con tres perfiles profesionales. En el área denominada contabilidad y administración (a) se consideraron a las personas cuyo perfil es definido por poseer estudios en curso o completos en ciencias económicas, con experiencia en administración de personal, contabilidad, impuestos o auditoría (Santos Stacco, 2006). El segundo perfil analizado fue el comercial, para la inclusión en el perfil de marketing y ventas (b) se consideraron a aquellas personas con estudios en curso o completos de comercialización, administración de empresas con orientación comercial, o experiencia suficiente en el área de ventas. El tercer perfil incluyó a individuos de recepción o atención al cliente (c), perfil poseído por personas interesadas en desempeñarse laboralmente en atención al cliente y con experiencia previa en dichas tareas (Marcaccio \& Moreno, 2013).

Lamentablemente se cuentan con limitadas referencias bibliográficas para hacer una descripción exhaustiva y unánime de los perfiles profesionales. Desde el punto de vista educativo, cada centro de estudio delimita el perfil de sus egresados de manera autónoma y desde 
el punto de vista laboral no se encuentran referencias respecto a la opinión de evaluadores y entrevistadores. En este trabajo se definen los perfiles profesionales, pero esta definición no puede considerarse exhaustiva.

Respecto al perfil contable algunos autores abogan por unificar el perfil (Martinez Gomez, 2007; Rodríguez, 1999), al menos en América. Sin embargo, en sus trabajos se refieren sólo a unificar conocimientos y evaluaciones, y a respetar normativas internacionales; no mencionan una definición única del perfil contable. Para trabajar en el campo contable se requieren algunos atributos como: interés en los negocios, conocimientos matemáticos y manejo de sistemas contables, habilidad para trabajar en equipo y honestidad. Aunque las personas idóneas pueden desempeñar algunas tareas del sector contable, para otras suele requerirse estudios de grado y posgrado.

Los profesionales en el sector de marketing buscan ideas para llamar la atención de los consumidores. Para lograr este objetivo se requiere el manejo de ordenadores y de redes sociales, entre otras variadas herramientas (Castelló Martínez, 2010). Las personas con perfil profesional de marketing diseñan, gestionan y evalúan planes y estrategias de comercialización destinados a satisfacer las demandas de clientes y consumidores. El trabajo en marketing se caracteriza por ser dinámico y cambiante.

La descripción del puesto de recepcionista suele incluir la responsabilidad de ser la persona de contacto y vinculación en una organización. Implica el trato con clientes, autoridades y otros colaboradores. Las habilidades comunicacionales son fundamentales en este puesto dado que deben comprender instrucciones, redactar correspondencia y elaborar presentaciones. Se requieren conocimientos matemáticos básicos y en algunos casos, es requerido el manejo de una segunda lengua. De acuerdo a las ofertas disponibles en el mercado laboral, no es necesario contar con educación formal en el área para desempeñar la tarea, aunque suele ser necesaria la experiencia o el entrenamiento. Un puesto de atención al cliente requiere habilidades similares, especialmente las referentes a la vinculación interpersonal. Deben afrontar el resolver conflictos, manejar las dificultades comunicacionales y emocionales de los clientes y el responder a sus necesidades.

\section{Perfiles profesionales y valores hacia el trabajo}

Ariel Knafo y Lilach Sagiv (2004) relacionaron el sistema de valores y el perfil ocupacional en trabajadores israelíes. En su investigación administraron la Escala de Perfiles Valorativos de Schwartz y clasificaron las 32 ocupaciones de los sujetos de la muestra de acuerdo a la tipología vocacional de Holland. Encontraron correlaciones negativas entre el valor Universalismo y la variable trabajo emprendedor, y observaron una correlación positiva entre los valores de Benevolencia y Universalismo con las ocupaciones sociales, así como una correlación negativa entre las ocupaciones sociales y los valores de Poder y Logro. La tipología artística correlacionó negativamente con el valor Conformidad, y la de investigador correlacionó positivamente con el valor Autodeterminación y negativamente con Tradición.

En una universidad de Turquía (Kaygin \& Gulluce, 2013) se encontraron relaciones significativas entre la elección de carrera y los valores personales. El estudio tenía entre sus propósitos determinar el valor más importante para cada una de las carreras estudiadas de la Facultad de Ciencias Económicas. En las tres carreras estudiadas se valoró en mayor medida el Logro.

Otro estudio basado en el modelo de valores universales de Schwartz (1992), comparó estudiantes de economía de negocios, con estudiantes en otras tres áreas de estudio con una orientación más social (Salud, Educación y Trabajo Social). Los primeros otorgaron menos importancia al valor Altruismo y al valor Universalismo y le otorgaron más peso a los valores Logro y Poder (Berings \& Adriaenssens, 2010). El mencionado estudio apoya la idea de que hay diferencias en la orientación ética de los estudiantes de ciencias económicas y los estudiantes de otros campos del conocimiento (Berings \& Adriaenssens, 2012), aunque no se haya podido determinar cuáles son las características del mecanismo causal.

En Argentina, se adaptaron anteriormente otros instrumentos para medir valores (Castro Solano \& Nader, 2006). María Martina Casullo y Alejandro Castro Solano (1997) presentaron un estudio en población civil y militar, en los que analizaron valores en diferentes contextos. Concluyeron que los valores tienen cierta consistencia transituacional, es decir, que no existen valores diferenciales según el contexto 
laboral o familiar, aunque existan algunas diferencias en el contexto social. A pesar del resultado se continuó trabajando con la evaluación situacional de valores. Adicionalmente, en ese trabajo se observó que los estudiantes de la carrera militar y los oficiales poseían sistemas de valores orientados hacia la Tradición, Conformidad y Seguridad. Los autores señalan que esos son los valores que se intentan socializar en la formación militar. En un estudio de Pablo Depaula (2012) se analizaron los valores y la performance en estudiantes militares. Se utilizó como instrumento la tercera parte del Cuestionario de Valores Contextualizados, VAL (Castro Solano, 2005; Casullo \& Castro Solano, 2004). La tercera parte del VAL mide valores relativos al trabajo y no se halló que los valores fueran predictores del desempeño.

Otros autores argentinos trabajaron con estudiantes de ciencias económicas. Graciela Filippi y Elena Zubieta (2003) compararon estudiantes de ciencias económicas y psicología de Buenos Aires y encontraron que, los estudiantes de ciencias económicas enfatizaban los valores relacionados con la Conservación combinados con metas de Logro y Poder, y los relacionados con la Autopromoción, la búsqueda del éxito personal y el dominio sobre otros. En otro trabajo compararon estudiantes argentinos y chilenos de ciencias económicas y empresariales. Los estudiantes chilenos priorizan el tener una mayor autonomía del pensamiento y acción, junto con obtener desafíos y retos, adicionalmente presentan mayor necesidad de poder, lo que resalta el prestigio y el estatus social e implica la dominación sobre personas y recursos. Existe un mayor énfasis de la individualidad, de la autopromoción, en los estudiantes chilenos en comparación con los argentinos (Zubieta \& Filippi, 2007). Respecto al género en ese trabajo se encontró heterogeneidad en los estudiantes de ciencias económicas según sexo. Depaula (2008) realizó un estudio sobre liderazgo y valores en el cual señala que las mujeres probablemente sean identificadas con el cuidado y la responsabilidad, la colaboración y la discusión democrática, además son más sensibles socialmente a relacionarse con los otros.

Kumar, Nonis y Thibodeaux (1997), encontraron que las diferencias existentes en los perfiles de valores evaluados mediante la escala de valores de Allport, Vernon y Lindzey en la década del sesenta no se evidencian, o están atenuadas, en la década del noventa. Señalan que el declive en los valores estéticos y religiosos en las mujeres estudiantes de negocios observado en su estudio había sido constatado también por otros investigadores. Harowitz (1987), señaló en una de sus publicaciones, que el altruismo es un valor que prácticamente ha desaparecido y que, el profesionalismo estricto, era el valor dominante en estudiantes a finales de los ochenta. La disminución de la fortaleza relativa de los valores religiosos y estéticos es tal vez resultado de que las estudiantes de negocios en los noventa estaban, en general, rompiendo con el estereotipo asociado a la mujer (Allport, Vernon \& Lindzey, 1970).

Aún existe controversia respecto a los valores e intereses y la elección de una carrera. Se han realizado estudios en el área de ciencias económicas, algunos autores (Frey \& Meier, 2003; Carter \& Irons, 1991; Gandal \& Roccas, 2002) consideran que las personas eligen carreras acordes con sus valores, esto se conoce como hipótesis de selección. Otros autores (Blais \& Young, 1999; Frank, Gilovich \& Regan, 1993) consideran que las personas modifican sus valores por influencia de la educación, esto se conoce como hipótesis de aprendizaje. Trabajos como el antes mencionado de Casullo y Castro Solano (1997), en que se hace referencia a la socialización de valores durante la carrera ,son compatibles con la hipótesis de aprendizaje.

En un estudio en laboratorio sobre la producción de un bien público, Marwell y Ames (1981) hallaron que los graduados en economía tendían a presentar mayor comportamiento tipo free rider ${ }^{1}$ que los no economistas. Los investigadores invitaron a economistas y no economistas a participar de un estudio experimental sobre decisiones de inversión. Los autores ofrecieron dos conjeturas sobre los motivos del comportamiento diferente en los estudiantes de economía. En primer lugar puede deberse a que, aquellos estudiantes a quienes les interesa la economía se pueden inclinar hacia las ciencias económicas; 0 alternativamente, los estudiantes de economía pueden adaptar su comportamiento, a lo largo del tiempo, para encajar en los axiomas de las teorías que ellos estudian. Este trabajo generó posteriores investigaciones. Algunos autores notaron que los economistas solían ver los test de laboratorio como test de rendimiento intelectual y los resultados no reflejaban sus valores.

\footnotetext{
1. Free rider: Concepto acuñado por Mancur Olson (1965), puede traducirse como "polizón" y define a alguien que suele sacar provecho del esfuerzo colectivo sin hacer la contribución correspondiente.
} 
Frey y Meier (2003) realizaron un estudio ecológico sobre la generosidad y el egoísmo en estudiantes de economía, administración de empresas y otras disciplinas. Trabajaron con más de 96.500 observaciones. Invitaron a estudiantes a contribuir, con cantidades pequeñas de dinero, con un fondo para créditos orientado a estudiantes en situación de necesidad y a otro fondo para estudiantes extranjeros. Recolectaron datos incluso antes de que los estudiantes asistieran a su primer curso. El resultado señaló que los estudiantes de administración de empresas tenían mayor egoísmo que los estudiantes de otras carreras, y esto no era producto de la formación recibida durante los estudios de grado. El estudio ofreció evidencia empírica en contra de la creencia de que los profesionales de ciencias económicas son más egoístas que otras personas y que eso se debe a la educación que reciben en economía. En el trabajo de Neil Gandal y Sonia Roccas (2002), los economistas le dieron mayor importancia al Logro y al Poder incluso antes de iniciar su formación.

Los estudiantes de economía suelen estar guiados más por el interés propio que aquellos que no estudian economía (Carter \& Irons, 1991). Además, le dan mayor prioridad a maximizar las ganancias que a las consideraciones sociales (Cipriani \& Zago, 2009), son menos generosos y menos cooperativos (Frank et al.,1993), parecen ser más egoístas (Frey \& Meier 2003, Meier \& Frey, 2004) y más dirigidos por el egoísmo y el individualismo (Wood, Longnecker, McKinney, \& Moore, 1988).

Se ha investigado la relación entre los valores laborales y otras variables como género (Walker, Tausky \& Oliver, 1982), ocupaciones (Moreno \& Marcaccio, 2013), diferencias culturales en población universitaria (Karakitapoğlu Aygün, Arslan \& Güney, 2008; Kumar \& Thibodeaux, 1998) y grupos generacionales (Cennamo \& Gardner, 2008).

\section{Metodología}

\section{Sujetos}

La muestra, no probabilística, incluyó a 425 sujetos ( $40 \%$ varones y $60 \%$ mujeres) que se encontraban buscando un cambio de empleo o un nuevo empleo, 243 de ellos aplicaron para trabajar en el área contable, 115 de ellos en marketing y 67 (en su totalidad mujeres) en el área de recepción.
El $39,3 \%$ de ellos poseía estudios terciarios o universitarios completos, el $44,4 \%$ poseía estudios terciarios o universitarios incompletos, el $15,8 \%$ estudios secundarios completos y secundarios incompletos el $0,5 \%$.

\section{Instrumentos}

El instrumento utilizado fue la Escala Argentina de Valores Relativos al Trabajo que comprende cuatro subescalas de cinco ítems cada una.

En un trabajo previo (Moreno \& Marcaccio, 2012) se presentó una versión de la Escala Revisada de Valores Relativos al Trabajo de Porto y Pilati (2010) traducida al castellano, abreviada y adaptada a la Argentina. En el mencionado trabajo se validó la prueba en una muestra de adolescentes $(N=333)$ y en una muestra de adultos $(\mathrm{N}=523)$. A diferencia de los autores originales, quienes procuraron construir una prueba de valores laborales que se ajustara a la teoría de valores generales de Schwartz, el trabajo se centró en determinar las dimensiones de los valores laborales basado en estudios empíricos. Los coeficientes alfa de Cronbach (1951) fueron satisfactorios en ambas muestras. Se encontraron cuatro dimensiones diferentes a las seis descriptas en la escala original para población brasileña. Los factores obtenidos luego del análisis factorial fueron interpretados como: Realización Personal, Altruismo, Reconocimiento Social y Aspiraciones Económicas. A continuación se describe cada factor.

- Realización Personal: Hace referencia a sentir que se tienen experiencias en el trabajo que favorecen el desenvolvimiento de las competencias individuales. Son sujetos que buscan desarrollarse personalmente, ser creativos y ser autores de su vida (Ítem 4: "Demostrar que soy competente en mi labor").

- Altruismo: Hace referencia a sentirse socialmente útil y procurar el bien común incluso a costa del propio. Son personas que necesitan sentir que su trabajo mejora la vida de otros seres humanos y beneficia a la sociedad en general (Ítem 1: "Ayudar a otros").

- Reconocimiento Social: Hace referencia a sentirse reconocido por el resto de la sociedad, ser famoso o admirado por la labor. Buscan prestigio (Ítem 8: "Ser reconocido por la profesión u ocupación que tengo").

- Aspiraciones Económicas: Hace referencia a sentir independencia financiera. Son individuos que buscan mejorar sus condiciones de 
vida mediante el trabajo y obtener logros materiales (Ítem 5: "Obtener estabilidad económica").

Los sujetos deben marcar el grado de acuerdo, en una escala de cuatro categorías ("Totalmente de acuerdo", "De acuerdo", "Un poco de acuerdo", "Para nada de acuerdo"), que tienen respecto a los valores relativos al trabajo que se detallan en 20 afirmaciones.

\section{Resultados}

En primer lugar se evaluaron los perfiles de los postulantes a cargos de ventas y comercialización y de los postulantes a empleos contables. Esta comparación se realizó con el total de las submuestras, dado que en ambas la proporción hombres-mujeres era semejante y cercana al $50 \%$ para cada categoría. Marketing 58 varones y 57 mujeres; Contabilidad 112 varones y 131 mujeres.

Se realizó un análisis multivariado de varianza (MANOVA) para determinar las diferencias de medias en las dimensiones de la prueba de valores hacia el trabajo (EVT), entre los sujetos de las submuestras de Marketing y Contabilidad (ver Tabla 1). En el análisis mul- tivariado de varianza se obtuvo un $\mathrm{F}$ Hotelling $(4,351)=4,53 p \leq .001$, es decir que las diferencias entre las medias de los valores hacia el trabajo según estas ocupaciones son significativas. Analizando los F univariados se observa que solamente el valor Realización personal explica dichas diferencias $(p=.002)$, siendo más jerarquizado por los sujetos de marketing. No se observan diferencias según sexo $(F=1.42$ $p=.22)$ ni interacción sexo - ocupación entre ambas submuestras $(\mathrm{F}=0.27 p=.89)$.

En segundo lugar evaluamos los perfiles de los postulantes a cargos de ventas y comercialización, de empleos contables y de recepcionistas en submuestras de mujeres, dado que para el cargo de recepcionista la totalidad de las postulantes eran mujeres. Recepcionistas $(n=67)$, Contabilidad ( $n=131)$, Marketing $(n=57)$.

Se realizó un análisis multivariado de varianza (MANOVA) para determinar las diferencias de medias en las dimensiones de la prueba de valores hacia el trabajo (EVT), entre los sujetos de las submuestras de Marketing, Recepcionistas y Contabilidad (ver Tabla 2).

En el análisis multivariado de varianza se obtuvo un F Hotelling $(8,496)=2,46 p \leq .01$, es

Tabla 1. Escala de Valores Relativos al Trabajo. Análisis Multivariado de Varianza (MANOVA), diferencias de medias y desvios típicos de los valores según perfil profesional

\begin{tabular}{|c|c|c|c|c|c|c|}
\hline \multirow{2}{*}{ Valores } & \multicolumn{2}{|c|}{$\begin{array}{c}\text { Marketing } \\
(n=115)\end{array}$} & \multicolumn{2}{|c|}{$\begin{array}{c}\text { Contabilidad } \\
(n=243)\end{array}$} & \multirow[t]{2}{*}{$\mathrm{F}$} & \multirow[t]{2}{*}{$p$} \\
\hline & Media & Desvío & Media & Desvío & & \\
\hline Reconocimiento Social & $290.3(4)$ & 54.2 & $288.5(4)$ & 52.1 & 0.09 & .75 \\
\hline Realización personal & $362.3(1)$ & 39.3 & $348.8(2)$ & 36.6 & 9.68 & $.002^{*}$ \\
\hline Altruismo & $320.0(3)$ & 56.7 & $327.2(3)$ & 51.6 & 1.49 & .22 \\
\hline Aspiraciones económicas & $359.1(2)$ & 45.3 & $356.3(1)$ & 40.1 & 0.39 & .53 \\
\hline
\end{tabular}

Tabla 2. Escala de Valores Relativos al Trabajo. Análisis Multivariado de Varianza (MANOVA), diferencias de medias y desvíos típicos de los valores según perfil profesional en muestra de mujeres

\begin{tabular}{lccccccccc}
\hline & \multicolumn{2}{c}{$\begin{array}{c}\text { Marketing } \\
\text { Valores }\end{array}$} & \multicolumn{2}{c}{$\begin{array}{c}\text { Contabilidad } \\
(\mathrm{n}=57)\end{array}$} & \multicolumn{2}{c}{$\begin{array}{c}\text { Recepcionista } \\
(\mathrm{n}=67)\end{array}$} & $\mathrm{F}$ & $p$ \\
\cline { 2 - 6 } & \multicolumn{2}{c}{ Media } & Desvío & Media & Desvío & Media & Desvío & \\
\hline Reconocimiento Social & $293.5(4)$ & 52.8 & $288.9(4)$ & 52.1 & $289.6(4)$ & 51.7 & 0.15 & .85 \\
Altruismo & $317.2(3)$ & 52.9 & $325.3(3)$ & 56.5 & $339.0(3)$ & 49.1 & 2.67 & .07 \\
Aspiraciones económicas & $365.2(1)$ & 37.3 & $357.9(1)$ & 39.5 & $356.0(1)$ & 40.3 & 0.94 & .39 \\
Realización personal & $362.7(2)$ & 32.7 & $346.7(2)$ & 39.5 & $344.5(2)$ & 41.2 & 4.23 & $.01^{*}$ \\
\hline
\end{tabular}

${ }^{*} p \leq .01$

Entre paréntesis figura el rango de las medias para cada una de las muestras. 
decir que las diferencias entre las medias de los valores hacia el trabajo según estas ocupaciones son significativas.

Analizando los $\mathrm{F}$ univariados se observa que, solamente el valor Realización Personal explica dichas diferencias $(p \leq .01)$, siendo más jerarquizado por los sujetos de marketing y en menor medida por las recepcionistas. En los contrastes multivariados del valor Realización Personal, son significativas las diferencias entre Marketing y Contabilidad y entre Marketing y Recepcionista. En ambos casos Marketing obtuvo mayores promedios.

\section{Discusión y conclusiones}

Este estudio incluyó a 425 sujetos (40\% varones y $60 \%$ mujeres) que se encontraban buscando un cambio de empleo o un nuevo empleo, 243 de ellos aplicaron para trabajar en el área contable, 115 de ellos en marketing y 67 (en su totalidad mujeres) en el área de recepción. A todos ellos se le administró la Escala Argentina de Valores Relativos al Trabajo (Moreno \& Marcaccio, 2012).

El trabajo en marketing se divide en dos grandes áreas: aquellas que implican un elevado nivel de análisis, como la realización de investigaciones de mercado, la realización y lectura de métricas, o la evaluación de presupuestos; y aquellas que favorecen el diseño creativo como el trabajo en desarrollo de producto, comunicación y medios. Las primeras tareas se encuentran directamente relacionadas con la toma de decisiones comerciales y las segundas con un trabajo en el que resalta el aporte personal y las ideas del sujeto. Es decir, existen reconocimientos personales sobre el desempeño. Para cumplir con cualquiera de estas tareas las personas con perfil profesional de marketing y ventas deben estar atentas a la interacción con consumidores, a empresas de la competencia y a los diferentes departamentos de la compañía. De este modo las tareas en marketing y ventas favorecen el desenvolvimiento de las competencias individuales. Es esperable que aquellos con perfil profesional de marketing y ventas valoren más la Realización Personal en el trabajo, que aquellos que deben realizar tareas en gran medida rutinarias o tareas en las que no se suele premiar el desempeño.
En el orden de rangos, las personas con perfil profesional de administración y contabilidad valoran más las Aspiraciones Económicas. Un valor que se encontró en segundo orden en el perfil profesional de marketing y ventas.

Habiéndose realizado un estudio que incorporaba un perfil profesional diferente, el de recepcionista, en una muestra compuesta por mujeres se confirma que las personas con perfil profesional de marketing y ventas valoran la Realización Personal en el trabajo más que otros perfiles profesionales.

Si bien no es significativo, se observa una tendencia en el perfil profesional de recepcionistas y atención al cliente a valorar en mayor medida el Altruismo, particularmente respecto del perfil profesional de marketing y ventas. Estas observaciones se ajustan un poco a los perfiles esperados y a estudios previos. En un estudio realizado utilizando la escala de valores interpersonales de Gordon (1993), las recepcionistas valorizaron en mayor medida tener un buen vínculo con el otro en el desempeño de su tarea y tendieron a adecuarse en mayor medida a las normas sociales. En el mencionado trabajo los profesionales de marketing valoraron más el Liderazgo, es decir, se orientaron a alcanzar puestos de mando con autoridad sobre las otras personas (Marcaccio \& Moreno, 2013).

Respecto a que no se encontraron en nuestro estudio diferencias significativas según sexo en las submuestras de contabilidad y marketing, es interesante tener en cuenta estudios longitudinales sobre perfiles de valores en estudiantes de escuela de negocios (Kumar et al., 1997). Es posible que la población en general asocie un grupo de valores con el género femenino, pero que las mujeres del área de contabilidad y marketing ya no los prioricen.

Resumiendo, los principales resultados fueron: 1) Existen diferencias en el valor Realización Personal entre los perfiles profesionales de Marketing y Contabilidad; 2) Las personas con perfil profesional de Marketing valoran más la Realización Personal que las personas con perfil profesional de Contabilidad y Recepción; 3) Sólo se encontraron diferencias en Realización Personal, una de las cuatro subescalas que componen la Escala Argentina de Valores Relativos al Trabajo. En las subescalas Reconocimiento Social, Altruismo y Aspiraciones Económicas no se observaron diferencias. 


\section{Referencias}

Allport, G., Vernon, P., \& Lindzey, G. (1970). Manual: Study of values. Chicago, II: Riverside Publishing.

Arnaz, J. A. (1981). Guía para la elaboración de un perfil del egresado. Revista de Educación Superior, 10(4), 1-7.

Arnaz, J. A. (1996). La planeación curricular. México: Trillas.

Beccaria, L. \& López, N. (1997). Sin trabajo. Las características del desempleo y sus efectos en la sociedad argentina. Buenos Aires: UNICEF y Losada.

Berings, D., \& Adriaenssens, S. (2010). Diversiteit in waarden en religiositeit in een hogeschool. Tijdschrift voor HogerOnderwijs, 28(2), 96-117.

Berings, D., \& Adriaenssens, S. (2012). The Role of Business Ethics, Personality, Work Values and Gender in Vocational Interests from Adolescents. Journal of Business Ethics, 106(3), 25-335. doi:10.1007/ s10551-011-0999-2

Blais, A. \& Young, R. (1999). Why do people vote? An experiment in rationality. Public Choice, 99(3), 9-55.

Carter, J. R., \& Irons, M. D. (1991). Are economists different, and if so, why? Journal of Economic Perspectives, 5, 171-177.

Castelló Martínez, A. (2010). Una nueva figura profesional: el Community Manager. Revista de la Red Académica Iberoamericana de Comunicación, 1, 74-97.

Castro Solano, A. \& Nader, M. (2006). La evaluación de los valores humanos con el Portrait Values Questionnaire de Schwartz. Interdisciplinaria, 23(2), 155 - 174.

Castro Solano, A. (2005). Técnicas de evaluación psicológica en los ambientes militares. Buenos Aires: Paidós.

Casullo, M. M. y Castro-Solano, A. (1997). Adaptación del MIPS en Argentina. En M. P. Sánchez-López y M. M. Casullo (Eds.), Los estilos de personalidad: Una perspectiva iberoamericana (pp. 233-265). Madrid y Buenos Aires: Miño y Dávila

Casullo, M. M. \& Castro Solano, A. (2003). La estructura de los valores humanos en población adulta argentina civil y militar. Una propuesta para su evaluación psicológica. Revista Digital Universitaria Colegio Militar de la Nación, 1(2). Recuperado de http://www. colegiomilitar. $\mathrm{mil}$. ar/rediu/numero.asp? numero $=2$

Casullo, M. M. \& Castro Solano, A. (2004). Valores humanos y contextos en población civil y militar. Acción Psicológica (UNED), 3(1), 21-30.

Cennamo, L. \& Gardner, D. (2008). Generational differences in work values, outcomes and person-organisation values fit, Journal of Managerial Psychology, 23(8), 891-906.

Cipriani, G. P., \& Zago, D. L. A. (2009). Natural born economists. Journal of Economic Psychology, 30, 455-468.

Cronbach, L. (1951). Coefficient alpha and the internal structure of test. Psychometrika, 16, 297-334

De Ibarrola, M. (2004). Paradojas recientes de la educación frente al mercado de trabajo y la inserción social. Buenos Aires: Instituto Internacional de Planeamiento de la Educación-RedEtis-ides.

Depaula, P. (2008). Actitudes hacia los líderes mujeres y valores en el trabajo. Un estudio comparativo entre población civil y militar. Revista Digital Universitaria Colegio Militar de la Nación, 6(18), 1-14. Recuperado de http://www.colegiomilitar.mil.ar/rediu/numero. asp?numero $=18$

Depaula, P. D. (2012). Predictores globales de la performance de estudiantes militares. Ciencias Psicológicas, 6(2), 135-148.
Díaz Barriga, F. (1993). Aproximaciones metodológicas al diseño curricular hacia una propuesta integral, en Tecnología y Comunicación Educativas, 21, 19-39.

Díaz Barriga, F. (1999). Elaboración del perfil profesional. En Díaz Barriga, F., Lule, M. L., Pacheco Pinzón, D., Rojas-Drummond, S., \& Saad Dayán, E. (Eds.), Metodología de Diseño Curricular para Educación Superior (pp. 85-104). México: Trillas.

Dose, J. J. (1997). Work Values: An Integrative Framework and Illustrative Application to Organizational Socialization. Journal of Occupational and Organizational Psychology, 70, 219-240.

Elizur, D. \& Koslowsky, M. (2001). Values and organizational commitment. International Journal of Manpower, 22(7), 593-599.

Feather, N. T., \& Rauter, K. A. (2004). Organizational citizenship behaviours in relation to job status, job insecurity, organizational commitment and identification, job satisfaction and work values. Journal of Occupational and Organizational Psychology, $77,81-94$

Filippi, G. \& Zubieta, E. (2003). Valores y Trabajo: un estudio con estudiantes universitarios. Anuario de investigaciones 2003. Instituto de Investigaciones, Facultad de Psicología, Universidad de Buenos Aires.

Frank, R. H., Gilovich, T. \& Regan, D. T. (1993). Does studying economics inhibit cooperation? Journal of Economic Perspectives, 7(2), 159-171.

Frey, B. S., \& Meier, S. (2003). Are political economists selfish and indoctrinated? Evidence from a natural experiment. Economic Inquiry, 41, 448-462.

Gandal, N. \& Roccas, S. (2002). Good neighbours/Bad citizens: Personal value priorities of economist. Discussion Paper $N^{\circ}$ 3660. London: Center for Economic Policy Research.

Glazman, R. \& de Ibarrola, M. (1978). Diseño de planes de estudio. México: CISE, UNAM.

Griffa, M. C. \& Moreno J. E. (2005). Claves para una Psicología del Desarrollo. Volumen II. Buenos Aires: Editorial Lugar.

Gordon, L. V. (1993). Survey of interpersonal values, SRA, Examiners manual ( $3^{\mathrm{a}}$ ed.). Londres: MacMillan.

Harowitz, H. L. (1987). Campus life: Undergraduate culture from the end of the century to present. Chicago, II: Chicago University Press.

Karakitapoğlu Aygün, Z., Arslan M. \& Güney, S. (2008), Work Values of Turkish and American University Students. Journal of Business Ethics, 80, 205-223. doi:10.1007/s10551-007-9413-5

Kaygin, E. \& Gulluce, A. C. (2013). The Relationship between Career Choice and Individual Values: A Case Study of a Turkish University. International Journal of Humanities and Social Science. 3(3), 119-134.

Knafo, A., \& Sagiv, L., (2004). Values and work environment: mapping 32 occupations. European Journal of Psychology of Education, 19(3), 255-273.

Kumar, K. \& Thibodeaux, M.S. (1998). Differences in Value Systems of Anglo-American and Far Eastern Students: Effects of American Business Education. Journal of Business Ethics, 17(3), 253-262.

Kumar, K., Nonis, S. A., \& Thibodeaux, M. S. (1997). Value profiles of business students in the 1960s and in the 1990s: a comparison. Journal of Education for Business. 72, 140-143.

Marcaccio, A. \& Moreno, J. E. (2013). Valores interpersonales y perfiles profesionales. Revista Argentina de Ciencias del Comportamiento, Suplemento, 278-279. 
Martinez Gomez, C. F. (2007), El deber ser de la contaduría pública en Colombia, Acierto Revista Académica y Científica De Uniremington, 2, 23-31.

Marwell, G. \& Ames, R. E. (1981). Economists free ride, does anyone else?: Experiments on the provision of public goods, IV. Journal of Public Economics 15, 295-310. doi:10.1016/0047-2727(81)90013-X

Meier, S. \& Frey, B. S. (2004). Do business students make good citizens? International Journal of the Economics of Business, 11(2), 141-163.

Moreno, J. E. \& Marcaccio, A. (2012). Escala de valores relativos al trabajo. Propiedades psicométricas de una versión en castellano revisada. Praxis. Revista de Psicología14(22), 65-78.

Moreno, J. E. \& Marcaccio, A. (2013, julio). Perfiles profesionales y valores relativos al trabajo. Comunicación presentada en el XXXIV Congreso Interamericano de Psicología. Sociedad Interamericana de Psicología. Brasilia, Brasil.

Olson, M. (1965). The Logic of Collective Action: Public Goods and the Theory of Groups. England: Harvard University Press.

Porto, J. B. \& Pilati, R. (2010). Escala Revisada de Valores Relativos ao Trabalho: EVT-R. Psicologia: Reflexão e Crítica, 23(1), 73-82.

Porto, J. B., \& Tamayo, A. (2003). Escala de valores do trabalho: EVT. Psicologia: Teoria e Pesquisa, 19(2), 145-152.

Porto, J. B., Pilati, R. \& Mendes Taixeira, M. L. (2006, setembro). Analisé Fatorial Confirmatória da Escala de Valores Relativos ao Trabalho. Comunicación presentada en $30^{\circ}$ Encontro da Anpad. Salvador, Brasil.

Pryor, R. (1979). In search of a concept: Work values. The Vocational Guidance Quarterly, 27, 250-258.

Rodríguez, L. (1999). Perfil del Contador Público en las Américas en el Siglo XXI. InterAmérica, 1(4).

Rokeach, M. (1973). The nature of human values. New York: Free Press.

Santos Stacco, J. (2006). "Hacia un nuevo perfil del graduado en ciencias económicas. ¿Cuál es la discusión que viene?". "Anuario 2005. Publicación de Trabajos Docentes" de la FCE de la UNPSJB.
Schein, E. H. (1978). Career dynamics: Matching individual and organizational needs. Reading, MA: Addison - Wesley.

Schein, E. H. (1993). Career anchors: Discovering your real values (Rev. Ed.). London: Pfeiffer.

Schwartz, S. H. (1992). Toward a universal psychological structure of human values. Journal of Personality and Social Psychology, 53, 550-562.

Siu, O. (2003). Job stress and job performance among employees in Hong Kong: The role of Chinese work values and organizational commitment. International Journal of Psychology, 38(6), 337-347.

Siu, O., Spector, P. E., Cooper, C. L., \& Lu, C. (2005). Work stress, self-efficacy, Chinese work values, and work well-being in Hong Kong and Beijing. International Journal of Stress Management, 12(3), 274-288.

Takase, M., Maude, P. \& Manias, E. (2005). Explaining nurses' work behaviour from their perception of the environment and work values. International Journal of Nursing Studies, 42(8), 889-898.

Taris, R. \& Feij, J. A. (2001). Longitudinal examination of the relationship between supplies-values fit and work outcomes. Applied Psychology: An International Review, 50(1), 52-80.

Villarreal, E. (1980). La planeación académica integral. Cuadernos de Planeación universitaria. México: UNAM.

Walker, J. E., Tausky, C \& Oliver, D. (1982). Men and women at work: Similarities and differences in work values within occupational groupings, Journal of Vocational Behaviour, 21 (1), 17-36.

Wood, J. A., Longnecker, J. G., McKinney, J. A., \& Moore, C. W. (1988). Ethical attitudes of students and business professionals: A study of moral reasoning. Journal of Business Ethics, 7(4), 249-357.

Zubieta, E. \& Filippi, G, (2007). Valores y creencias asociadas al trabajo en estudiantes universitarios de Argentina y Chile. SUMMA Psicológica UST, 4(2), 81-98.

Para citar este artículo:

Moreno, J. E., \& Marcaccio, A. (2014). Perfiles profesionales y valores relativos al trabajo. Ciencias Psicológicas VIII (2): 129 - 138. 